Proc. Indian Acad. Sci. (Math. Sci.) Vol. 113, No. 2, May 2003, pp. 189-193.

Printed in India

\title{
The Weierstrass-Enneper representation using hodographic coordinates on a minimal surface
}

\author{
RUKMINI DEY \\ School of Mathematics, Harish-Chandra Research Institute, Allahabad 211 019, India \\ E-mail: rkmn@mri.ernet.in
}

MS received 19 June 2002; revised 10 October 2002

\begin{abstract}
In this paper we obtain the general solution to the minimal surface equation, namely its local Weierstrass-Enneper representation, using a system of hodographic coordinates. This is done by using the method of solving the Born-Infeld equations by Whitham. We directly compute conformal coordinates on the minimal surface which give the Weierstrass-Enneper representation. From this we derive the hodographic coordinate $\rho \in D \subset \mathbb{C}$ and $\sigma$ its complex conjugate which enables us to write the Weierstrass-Enneper representation in a new way.
\end{abstract}

Keywords. Minimal surface; hodographic coordinates; conformal coordinates; Weierstrass-Enneper representation

\section{Introduction}

Minimal surfaces are most commonly known as surfaces which have the minimum area amongst all other surfaces spanning a given closed curve in $\mathbb{R}^{3}$. Geometrically, the definition of a minimal surface is that the mean curvature $H \equiv 0$ at every point of the surface. If locally one can write the minimal surface in $\mathbb{R}^{3}$ as $(x, y, \phi(x, y))$ the minimal surface equation $H \equiv 0$ is equivalent to

$$
\left(1+\phi_{y}^{2}\right) \phi_{x x}-2 \phi_{x} \phi_{y} \phi_{x y}+\left(1+\phi_{x}^{2}\right) \phi_{y y}=0 .
$$

There exists a choice of conformal coordinates $(u, v) \in \Omega \subset \mathbb{R}^{2}$ so that the surface $X(u, v)=(x(u, v), y(u, v), \phi(u, v)) \in \mathbb{R}^{3}$ satisfying the minimal surface equation is given as follows [1]:

$$
\left|X_{u}\right|^{2}=\left|X_{u}\right|^{2}, \quad\left\langle X_{u}, X_{v}\right\rangle=0, \quad \Delta_{(u, v)} X=0 .
$$

The general solution of such an equation is called the local Weierstrass-Enneper representation. Let $D$ be a simply connected domain in $\mathbb{C}, f$ an analytic function and $g$ a meromorphic function on $D$. Then,

$$
X(\tau)=\Re \int_{\tau_{0}}^{\tau} \Phi \mathrm{d} \zeta
$$

where

$$
\Phi=\left(\left(1-g^{2}\right) f, i\left(1+g^{2}\right) f, 2 f g\right)
$$


is a conformal immersion of $D$ into $\mathbb{R}^{3}$ which is minimal [6]. The immersion is regular provided that wherever $g$ has a pole of order $m, f$ has a zero of at least order $2 m$. Moreover, $g$ is the stereographic projection of the Gauss map.

There is a simpler representation, valid away from the umbilical points of the surface. Let $w=g(\tau)$ and $R(w)=f(w)[\mathrm{d} g / \mathrm{d} \tau]^{-1}$. The Gaussian curvature of the surface is $K=$ $-4|R(w)|^{-2}\left(1+|w|^{2}\right)^{-4}$. Away from the umbilical points where $K$ vanishes, $\mathrm{d} g / \mathrm{d} \tau \neq$ 0 and $R(w)$ has no pole. Thus in the neighborhood of a nonumbilic interior point, any minimal surface can be represented in terms of $w$ as follows [5]:

$$
\begin{aligned}
& x(\zeta)=x_{0}+\Re \int_{\zeta_{0}}^{\zeta}\left(1-w^{2}\right) R(w) \mathrm{d} w, \\
& y(\zeta)=y_{0}+\Re \int_{\zeta_{0}}^{\zeta} i\left(1+w^{2}\right) R(w) \mathrm{d} w, \\
& \phi(\zeta)=\phi_{0}+\Re \int_{\zeta_{0}}^{\zeta} 2 w R(w) \mathrm{d} w .
\end{aligned}
$$

In this semi-expository paper we show that a system of hodographic coordinates gives us the local Weierstrass-Enneper representation of a minimal surface. Our method provides an easy way of calculating conformal coordinates if the formula for the graph of the minimal surface is given, locally. This is not given in the standard text books. Hodographic coordinates are a natural concept in fluid mechanics where velocity fields play the role of independent variables. It was mentioned in the context of minimal surfaces first in [3] and was used in the context of Born-Infeld equations in [7]. If one replaces $y$ by iy in the Born-Infeld equations, one obtains the minimal surface equation. Thus it is natural to expect a general solution for the minimal surface by following Whitham's method [7] for the Born-Infeld equation. Finally we derive the hodographic coordinates $\rho, \sigma$, complex conjugates of each other, which enables us to write the Weierstrass representation in a new way.

\section{Hodographic coordinates and Weierstrass-Enneper representation}

In the height representation of the minimal surface, or Monge gauge, one writes the minimal surface equation in $\mathbb{R}^{3}$ as in (1).

Introducing the complex coordinates $z=x+i y$ and $\bar{z}=x-i y$, we define $u=\phi_{\bar{z}}$ and $v=\phi_{z}=\bar{u}$ to reduce the second-order differential equation (1) to a pair of first-order equations:

$$
\begin{aligned}
& u_{z}-v_{\bar{z}}=0, \\
& v^{2} u_{\bar{z}}-(1+2 u v) u_{z}+u^{2} v_{z}=0 .
\end{aligned}
$$

The hodograph transformation interchanges the dependent and independent variables $(z, \bar{z}) \leftrightarrow(u, v)$. To do this we use

$$
\left[\begin{array}{ll}
z_{u} & z_{v} \\
\bar{z}_{u} & \bar{z}_{v}
\end{array}\right]\left[\begin{array}{ll}
u_{z} & u_{\bar{z}} \\
v_{z} & v_{\bar{z}}
\end{array}\right]=\left[\begin{array}{ll}
1 & 0 \\
0 & 1
\end{array}\right]
$$


and find

$$
\begin{aligned}
& \bar{z}_{v}-z_{u}=0, \\
& v^{2} z_{v}+(1+2 u v) \bar{z}_{v}+u^{2} \bar{z}_{u}=0 .
\end{aligned}
$$

Note that we have transformed the nonlinear partial differential equations for $u$ and $v$ into linear partial differential equations for $z$ and $\bar{z}$. Thus, it should come as no surprise that the minimal surface equation has a linear representation. Following [7], we introduce the new variables $\zeta=(\sqrt{1+4 u v}-1) /(2 v), \bar{\zeta}=(\sqrt{1+4 u v}-1) /(2 u)$ to facilitate our solution. The inverse of this transformation is $u=\zeta /(1-\zeta \zeta)$.

\section{PROPOSITION 2.1.}

In these new coordinates the eqs (8) and (9) are greatly simplified:

$$
\zeta^{2} \bar{z}_{\zeta}+z_{\zeta}=0
$$

Proof. Using the inverse transformation $u=\zeta /(1-\zeta \zeta), v$ its complex conjugate, and the equalities $z_{\zeta}=z_{v} v_{\zeta}+z_{u} u_{\zeta}$ and $\bar{z}_{\zeta}=\bar{z}_{v} v_{\zeta}+\bar{z}_{u} u_{\zeta}$, we obtain $\bar{z}_{\zeta}=\left(\bar{z}_{v} \bar{\zeta}^{2}+\bar{z}_{u}\right) /\left(1-|\zeta|^{2}\right)^{2}$ and $z_{\zeta}=\left(z_{v} \zeta^{2}+z_{u}\right) /\left(1-|\zeta|^{2}\right)^{2}$. Then eq. (10) is equivalent to

$$
\frac{\zeta^{2} \bar{z}_{u}+\bar{z}_{v}(\zeta \zeta)^{2}+z_{u}+z_{v} \zeta^{2}}{(1-\zeta \zeta)^{2}}=0
$$

Using the expression for $u$ and $v$ in terms of $\zeta$ and $\zeta$ we rewrite (9) as

$$
\frac{\zeta^{2} z_{v}+\left(1+(\zeta \zeta)^{2}\right) \bar{z}_{v}+\zeta^{2} \bar{z}_{u}}{(1-\zeta \zeta)^{2}}=0
$$

Substracting (12) from (11) and using (8) we get zero.

Thus eqs (8) and (9) are equivalent to eq. (10).

Now taking derivative of eq. (10) with respect to $\zeta$, we obtain

$$
\zeta^{2} \bar{z}_{\zeta \zeta}+z_{\zeta \zeta}=0
$$

Using (13) and its complex conjugate we immediately obtain $\bar{z}_{\zeta \zeta}=z_{\zeta \zeta}=0$ from which it follows that

$$
\bar{z}=\bar{z}_{0}+F(\zeta)+G(\zeta)
$$

Using (10) we find

$$
\zeta^{2} F^{\prime}(\zeta)+\bar{G}^{\prime}(\zeta)=0
$$

so

$$
\bar{z}=\bar{z}_{0}+F(\zeta)-\int_{\zeta_{0}}^{\zeta} \bar{\omega}^{2} \bar{F}^{\prime}(\bar{\omega}) \mathrm{d} \bar{\omega}
$$


Moreover, we have $\phi_{\zeta}=\phi_{\bar{z}} \bar{z}_{\zeta}+\phi_{z} z_{\zeta}=u F^{\prime}(\zeta)-v \zeta^{2} F^{\prime}(\zeta)=\zeta F^{\prime}(\zeta)$ so that

$$
\phi=\phi_{0}+\int_{\zeta_{0}}^{\zeta} \omega F^{\prime}(\omega) \mathrm{d} \omega+\int_{\zeta_{0}}^{\zeta} \bar{\omega} \bar{F}^{\prime}(\bar{\omega}) \mathrm{d} \bar{\omega} .
$$

It is straightforward to check that the coordinates $\zeta_{1}=\mathfrak{R} \zeta$ and $\zeta_{2}=\mathfrak{I} \zeta$ are isothermal so that $\left|X_{\zeta_{1}}\right|^{2}=\left|X_{\zeta_{2}}\right|^{2}$ and $\left\langle X_{\zeta_{1}}, X_{\zeta_{2}}\right\rangle=0$.

Rewriting (16) for $x$ and $y$, we have

$$
\begin{aligned}
& x(\zeta)=x_{0}+\Re \int_{\zeta_{0}}^{\zeta}\left(1-\omega^{2}\right) F^{\prime}(\omega) \mathrm{d} \omega, \\
& y(\zeta)=y_{0}+\Re \int_{\zeta_{0}}^{\zeta} i\left(1+\omega^{2}\right) F^{\prime}(\omega) \mathrm{d} \omega, \\
& \phi(\zeta)=\phi_{0}+\Re \int_{\zeta_{0}}^{\zeta} 2 \omega F^{\prime}(\omega) \mathrm{d} \omega .
\end{aligned}
$$

Letting $F^{\prime}(\omega)=R(\omega)$, eqs (18)-(20) are the Weierstrass-Enneper representation away from the umbilical points of the surface [5]. At an umbilical point, the Gaussian curvature $K$ vanishes so $\phi_{z z} \phi_{\bar{z} \bar{z}}-\phi_{z \bar{z}}^{2}=u_{\bar{z}} v_{z}-u_{z} v_{\bar{z}}=0$, precisely where (7) has no solution.

If $F^{\prime}(\zeta) \neq 0$, we can locally introduce new variables $\rho=F(\zeta), \sigma=G(\eta)$. Locally the inverse exists when the Gaussian curvature is finite. This follows from the fact that $K=-4 /\left(\left|\frac{\partial \rho}{\partial \zeta}\right|^{2}\left(1+|\zeta|^{2}\right)^{4}\right)$. If inverse exists, $(x, y, \phi)$ can be written as

$$
\begin{aligned}
& x=\frac{\rho+\sigma}{2}-\frac{1}{2} \int_{\rho_{0}}^{\rho}\left(F^{-1}(\tilde{\rho})\right)^{2} \mathrm{~d} \tilde{\rho}-\frac{1}{2} \int_{\sigma_{0}}^{\sigma}\left(G^{-1}(\tilde{\sigma})\right)^{2} \mathrm{~d} \tilde{\boldsymbol{\sigma}}, \\
& y=\frac{\sigma-\rho}{2 i}-\frac{1}{2 i} \int_{\rho_{0}}^{\rho}\left(F^{-1}(\tilde{\rho})\right)^{2} \mathrm{~d} \tilde{\rho}+\frac{1}{2 i} \int_{\sigma_{0}}^{\sigma}\left(G^{-1}(\tilde{\boldsymbol{\sigma}})\right)^{2} \mathrm{~d} \tilde{\boldsymbol{\sigma}}, \\
& \phi=\int_{\rho_{0}}^{\rho} F^{-1}(\tilde{\rho}) \mathrm{d} \tilde{\rho}+\int_{\sigma_{0}}^{\sigma} G^{-1}(\tilde{\sigma}) \mathrm{d} \tilde{\sigma},
\end{aligned}
$$

where $\zeta=F^{-1}(\rho)=\partial \phi / \partial \rho$ and $\zeta=G^{-1}(\sigma)=\partial \phi / \partial \sigma$. Thus

$$
\begin{aligned}
& x=\frac{\rho+\sigma}{2}-\frac{1}{2} \int_{\rho_{0}}^{\rho}\left(\phi_{\tilde{\rho}}\right)^{2} \mathrm{~d} \tilde{\rho}-\frac{1}{2} \int_{\sigma_{0}}^{\sigma}\left(\phi_{\tilde{\sigma}}\right)^{2} \mathrm{~d} \tilde{\sigma}, \\
& y=\frac{\sigma-\rho}{2 i}-\frac{1}{2 i} \int_{\rho_{0}}^{\rho}\left(\phi_{\tilde{\rho}}\right)^{2} \mathrm{~d} \tilde{\rho}+\frac{1}{2 i} \int_{\sigma_{0}}^{\sigma}\left(\phi_{\tilde{\sigma}}\right)^{2} \mathrm{~d} \tilde{\sigma}, \\
& \phi=\phi(\rho)+\phi(\sigma) .
\end{aligned}
$$

This decomposition is different from that of the isothermal coordinates $\zeta$ and $\zeta$. If $\rho=\rho_{1}+i \rho_{2}$, then it can be shown that $\left|X_{\rho_{1}}\right|=\left|X_{\rho_{2}}\right|$ and $\left\langle X_{\rho_{1}}, X_{\rho_{2}}\right\rangle=0$. Thus $\rho_{1}, \rho_{2}$ are the isothermal coordinates. The $\zeta$ system and the $\rho$ system are related by a conformal map, $F(\zeta)$ and its inverse.

The geometric meaning of $\phi_{\rho}$ is as follows: The unit normal to the surface in the $\rho$ system is given by

$$
N=\frac{X_{\rho_{1}} \times X_{\rho_{2}}}{\left|X_{\rho_{1}} \times X_{\rho_{2}}\right|}=\left(\frac{2 \operatorname{Re} \phi_{\rho}}{1+\left|\phi_{\rho}\right|^{2}}, \frac{2 \operatorname{Im} \Phi_{\rho}}{1+\left|\phi_{\rho}\right|^{2}}, \frac{\left(\left|\phi_{\rho}\right|^{2}-1\right)}{1+\left|\phi_{\rho}\right|^{2}}\right) .
$$


Thus $\phi_{\rho}$ is the stereographic projection of the Gauss map [6].

\section{An example}

We consider the helicoid, $\phi=\tan ^{-1}(y / x)$. We have $u=i / 2 \bar{z}$ and $v=-i / 2 z$ so that

$$
\begin{aligned}
& \bar{z}=\frac{i}{2 u}=\frac{i}{2}\left[\frac{1}{\zeta}-\eta\right], \\
& z=\frac{-i}{2 v}=\frac{-i}{2}\left[\frac{1}{\eta}-\zeta\right],
\end{aligned}
$$

where $\eta=\zeta$, from which it follows that the hodographic coordinates are $\rho=F(\zeta)=i / 2 \zeta$ and $\sigma=G(\eta)=-i / 2 \eta$. The solution $\phi$ is

$$
\begin{aligned}
\phi & =-\frac{i}{2} \ln \zeta+\frac{i}{2} \ln \eta \\
& =\frac{-i}{2} \ln \left[\frac{z}{\bar{z}}\right] \\
& =\tan ^{-1}\left(\frac{y}{x}\right) .
\end{aligned}
$$

Finally, note that $R(\omega)=F^{\prime}(\omega)=-i / 2 \omega^{2}$ is the standard result for the helicoid [5].

\section{Acknowledgement}

I would like to thank Professor Randall Kamien, for suggesting this problem to me and for useful discussions. I would like to thank Dr. Rajesh Gopakumar and Dr. Abhijit Mukherjee for their helpful comments.

\section{References}

[1] Dierkes U, Hildebrandt S, Kuster A and Wohlrab O, Minimal surfaces I (1992) (Springer-Verlag)

[2] Do Carmo M, Differential geometry of curves and surfaces (1976) (Prentice Hall)

[3] Kamien R D, Decomposition of the height function of Scherk's first surface, Appl. Math. Lett. 14 (2001) 797

[4] Kamien R D and Lubensky T C, Minimal surfaces, screw dislocations and twist grain boundaries, Phys. Rev. Lett. 82 (1999) 2892

[5] Nitsche J C C, Lectures on minimal surfaces (1989) (Cambridge University Press) vol. 1

[6] Osserman R, Survey of minimal surfaces (1986) (New York: Dover Publications)

[7] Whitham G B, Linear and nonlinear waves (1999) (John Wiley and Sons) 